\title{
Collaborative QoS Architecture between DiffServ and 802.11e Wireless LAN
}

\author{
Seyong Park ${ }^{*}$, Kyungtae Kim ${ }^{*}$, Doug C. Kim ${ }^{*}$, Sunghyun Choi ${ }^{+}$, Sangjin Hong ${ }^{*}$ \\ *Mobile Systems Design Laboratory \\ Department of Electrical and Computer Engineering \\ State University of New York at Stony Brook \\ Stony Brook, NY 11794-2350, USA \\ \{seyong,kyungtae,dougkim,snjhong\}@ece.sunysb.edu \\ ${ }^{+}$Multimedia \& Wireless Networking Laboratory \\ School of Electrical Engineering \\ Seoul National University \\ Seoul 151-744, KOREA \\ schoi@snu.ac.kr
}

\begin{abstract}
As the multimedia applications such as Voice over IP (VoIP) and Audio/Visual (AV) streaming across the Internet emerge, many are working on the network architecture to extend such applications to the wireless networking domain. The emerging IEEE 802.11e Quality-ofService (QoS)-enabled Wireless LAN (WLAN) is considered a strong candidate for the air interface for such multimedia applications thanks to the IP-centric network paradigm along with its inherent high-speed transmission capability. This paper provides an integrated wired/wireless network architecture interfacing QoS between user level traffic over IP using Differentiated Service (DiffServ) and transport level traffic using IEEE 802.11e WLAN. Our study investigates the correlations in end-to-end traffic management between DiffServ and 802.11e, and presents the hierarchical QoS signaling interface between DiffServ and 802.11e, in terms of traffic classifying, shaping and policing.
\end{abstract}

Keywords- IEEE 802.11, IEEE 802.11e, WLAN, DiffServ, Quality-of-Service (QoS)

\section{INTRODUCTION}

As broadband Internet access becomes pervasive, many multimedia applications such as VoIP, AOD and VOD have been serviced via Internet Service Providers (ISPs). In order to maintain a required performance for such applications, Internet network systems have been facilitating Quality-of-Service (QoS) to enable the network systems to prioritize traffic according to the application's service types. To our best knowledge, the IETF Differentiated Service (DiffServ) [10] is at the center of such efforts. As the portable devices such as laptops and palmtops become more and more popular, the interest to have a level of services similar to those available from the conventional wired networks using those portable devices without wires is growing very fast these days.

On the other hand, the IEEE 802.11 Wireless LAN (WLAN) has become a prevailing broadband wireless technology in recent years. Today, the IEEE 802.11 WLAN is considered a "wireless Ethernet" by virtue of its best effort service provisioning based on Ethernet-like medium access control (MAC) protocol and up to 54 Mbps transmissions rates. However, the 802.11 WLAN is also evolving to support QoS currently, and a new QoSenabled MAC called IEEE 802.11e is emerging [3].

For the multimedia applications to work properly, the end-to-end QoS should be in place. That is, for example, neither IEEE 802.11e WLAN alone nor the DiffServ without a proper QoS support from the air interface is enough for the full-fledged wireless multimedia applications. We, in this paper, consider an end-to-end QoS architecture across wired WAN, wired LAN, and wireless LAN, which are based on DiffServ, IEEE 802.1D/Q, and IEEE 802.11e, respectively.

This paper explores DiffServ, 802.11e and 802.1D/Q architectures in Sections III and IV. Section V presents two methodologies coordinating traffic QoS between DiffServ and $802.11 \mathrm{e}$ over the air interface, between 802.11e and 802.1D in Ethernet LAN interface, and DiffServ Per Hop Behavior (PHB) traffic control in IP WAN. Finally, this paper concludes with Section VI.

\section{SYSTEM MODELING AND BACKGROUND}

From the overall network service perspective, QoS should provide end-to-end traffic control so that users' applications can be properly served according to the allowable quality requirements such as latency, jitters and packet loss rate. To comply with the service quality requirements, user level traffic of the applications should coordinate QoS traffic control with transport level QoS at the network interfaces.

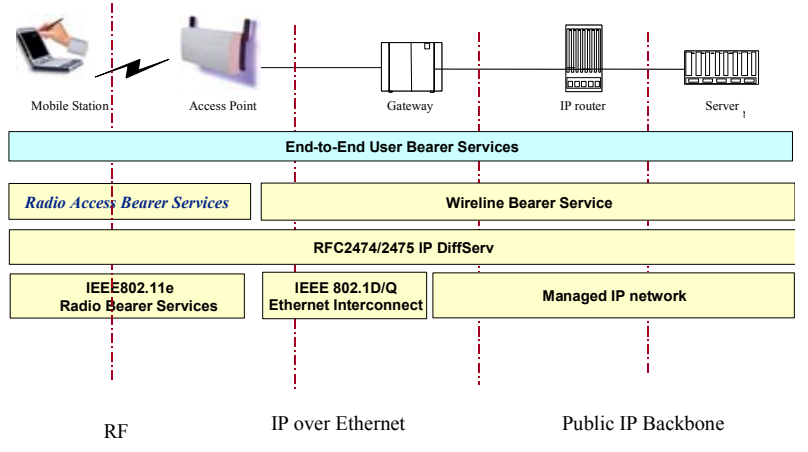

Fig. 1. End-to-end QoS network structures

As illustrated in Fig. 1, the user traffic QoS specifies end-to-end network traffic delay, jitter and policing. Since most data service accessing remote servers carries user traffic through multiple heterogeneous networking environments, the user level QoS should be decomposed into each network interface segment as follows:

(a) Radio Access network: air interface between mobile station (STA) and access point (AP) defined by IEEE 802.11e. Under the prioritized QoS paradigm, the $802.11 \mathrm{e}$ provides differentiated channel access to traffic with 8 different priority levels.

(b) Ethernet LAN: Ethernet between AP and Gateway terminating subnet traffic including traffic through other MAC bridges. $802.1 \mathrm{D} / \mathrm{Q}$ defines the QoS mechanisms, which can be used in the Ethernet LAN. Similar to the DiffServ, it also classifies the traffic 
type and prioritizes upon the traffic class at the bridge.

(c) Managed IP WAN: wireline interface managed by network service providers such as Competitive Local Exchange Carrier (CLEC) and Incumbent Local Exchange Carrier (ILEC). The IP WAN is normally managed by Service Level Agreement (SLA). User traffic's DiffServ parameters can be directly reflected into the IP routers to be prioritized over other traffic.

The IEEE 802.11e is an emerging standard for QoSenabled MAC for the popular IEEE 802.11 WLAN. As of today, the $802.11 \mathrm{e}$ standardization is at the final stage [3], and there have been some reports about the utility of this emerging technology, e.g., [6][7]. However, these reports have been focused on QoS issues at the layer-2 single hop i.e., wireless link between a STA and an AP. Since most user traffic in application layer traverses multiple networks as depicted in Fig. 1, the end-to-end QoS also becomes crucial to offer various network services in the real networks. Even though there was also a paper dealing with an end-to-end QoS paradigm based IntServ over IEEE 802.11e WLAN [8], to our best knowledge, today DiffServ is considered a dominant QoS protocol in the network layer that supports across different network interfaces. However, in wireless and wired LAN, DiffServ cannot function to traffic control for QoS. Instead, IEEE 802.11e and IEEE 802.1D are QoS in MAC layer. Since 802.11e and 801.1D are non-DiffServ domains, end-to-end QoS environment cannot be properly provided unless these different QoS techniques are coordinated under common QoS specifications.

Our study is to propose a methodology translating the DiffServ Per Hop Behavior (PHB) to the 802.11e and 802.1D.

\section{DIFFERENTIATED SERVICE (DIFFSERV)}

The DiffServ architecture [10] provides a scalable QoS framework for service providers that offer differentiated services under heterogeneous network environment. Each IP datagram carries a Differentiated Service CodePoint (DSCP) value in its Differentiated Service (DS) field, which supercedes the IPv4 Type of Service (TOS) octet and IPv6 Traffic Classifier octet. A network node supporting DiffServ consists of functional elements including Per Hop forwarding behavior, traffic classification, and conditioning function. Depending on the type of the network interface, normally, different functional elements are actually used. That is, as presented in the following subsections, the traffic classification and conditioning are conducted at the ingress network node while the PHB forwarding is conducted at the intermediate network node.

\section{A. Ingress network node}

Ingress network nodes such as edge routers or gateways perform the packet classification and conditioning. A packet classifier is a logical function to select packets based on packet information via either Behavior Aggregate (BA) Classifier searching the DSCP value or Multi-Field (MF) Classifier searching multiple fields including source and destination addresses, DSCP, port ID, etc. When a packet is classified, it is forwarded to the traffic conditioner that consists of meter, market, shaper and dropper. Meter measures the packet stream based on the traffic profile defined by Traffic
Conditioning Agreement (TCA) that is typically part of network provisioning contract. Packet marker sets the DSCP value given by classifier and meter. Shaper and Dropper function to police the packet traffic according to the temporal traffic conditions.

\section{B. Intermediate network node}

An intermediate network node performs a Per Hop Behavior (PHB), which is a logical instantiation performing traffic forward behavior. The forward behavior normally follows the traffic resource allocation per link based on the priority defined in DSCP. The traffic resource is determined based on packet loss rate, propagation delay and jitter. The PHB should maintain a mapping table between DSCP and forward behavior functions. DiffServ defines four available standard PHBs:

- Default PHB [9]

- Class-Selector PHB[9]

- Assured Forwarding (AFxy) PHB [12]

- Expedited Forwarding (EF) PHB [11]

The Default PHB is a best-effort service traffic that never assures any traffic parameters such as delay, jitter and throughput. It is typically implemented using the first-in first-out (FIFO) queuing. Most non-real-time data traffic is served using this PHB. The Class-Selector PHB is used for the backward compatibility with the existing IP precedence scheme current used in the IP network. The AF PHB is defined to support controlled traffic management at the QoS network node. The AF PHB is expressed as "AFxy." The ' $\mathrm{x}$ ' represents AF class number and ' $y$ ' represents the drop precedence. For example, in case of traffic congestion, AF13 should be dropped before AF12. AF classes are typically controlled by prioritized queues and traffic bandwidth following the Service Level Agreement (SLA). The EF PHB is the highest traffic class supporting for the real-time traffic such as VoIP or real-time video that require low delay and jitter. PHB may also include a specification for traffic conditioning described in Section III.A.

\section{802.11E AND 802.1D FOR QOS-ENABLED LANS}

\section{A. Legacy 802.11 WLAN}

The 802.11 MAC protocol [1] consists of two coordination functions, which are a mandatory distributed coordination function (DCF) built on Carrier Sense Multiple Access with Collision Avoidance (CSMA/CA) and an optional point coordination functions (PCF) built on a poll-and-response protocol. Today, most 802.11 devices implement the mandatory DCF mode only. The DCF functions traffic control based on non-preemptive service (i.e., FIFO). When the MAC frame arrives at the queue, it shall wait until the channel becomes idle, and defers another fixed time interval, called DCF InterFrame Space (DIFS), to avoid the potential collision with other network nodes. When the channel stays idle for the DIFS interval, it starts the random backoff (BO) counter. When the BO counter expires, the frame is transmitted over the air. When a frame arrives at an empty queue and the medium has been idle longer than the DIFS time interval, the frame is transmitted immediately.

Each STA maintains a contention window $(\mathrm{CW})$, which is used to select the random backoff count. The backoff 
count is determined as a pseudo-random integer drawn from a uniform distribution over the interval [0,CW]. If the channel becomes busy during a backoff process, the backoff is suspended. When the channel becomes idle again, and stays idle for an extra DIFS time interval, the backoff process resumes with the suspended backoff counter value. The timing of DCF channel access is illustrated in Fig. 2.

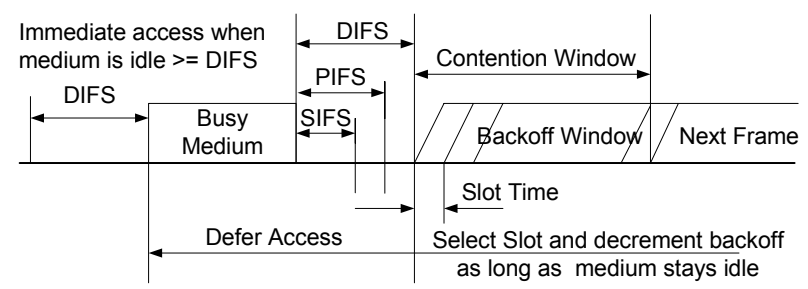

Fig. 2. 802.11 DCF Channel Access

\section{B. QoS-Enabled 802.11e WLAN}

The IEEE 802.11e defines a single coordination function, called the hybrid coordination function (HCF), for the QoS provisioning. The HCF combines functions from the DCF and PCF with some enhanced QoS-specific mechanisms and QoS data frames in order to allow a uniform set of frame exchange sequences to be used for QoS data transfers. The HCF uses a contention-based channel access, called the enhanced DCF (EDCF), which operates concurrently with a controlled channel access based on a poll-and-response protocol. In this paper, only EDCF is enclosed in the scope.

The emerging EDCF is designed to provide differentiated, distributed channel accesses for frames with 8 different priorities (from 0 to 7 ) by enhancing the legacy DCF. Each frame from the higher layer arrives at the MAC along with a specific priority value. Each QoS data frame also carries its priority value in the MAC frame header. In the context of the $802.11 \mathrm{e}$, the priority value is called Traffic Category Identification (TCID). An 802.11e STA shall implement four access categories (ACs), where an AC is an enhanced variant of the DCF with a single FIFO queue, as shown in Fig. 4. Each frame arriving at the MAC with a priority is mapped into an $\mathrm{AC}$ as shown in TABLE I. Note the relative priority of priority 0 is placed between 2 and 3 . This relative priority is rooted from IEEE 802.1D bridge specification [4].

TABLE I. TCID TO ACCESS CATEGORY MAPPINGS

\begin{tabular}{ccc}
\hline TCID / Priority & Access Category & Traffic Type \\
\hline 1 & 0 & Best Effort \\
2 & 0 & Best Effort \\
0 & 0 & Best Effort \\
3 & 1 & Video Probe \\
4 & 2 & Video \\
5 & 2 & Video \\
6 & 3 & Voice \\
7 & 3 & Voice \\
\hline
\end{tabular}

Basically, an AC uses AIFSD [AC], CWmin[AC], and CWmax[AC] instead of DIFS, CWmin, and CWmax, of the DCF, respectively, for the contention to transmit a frame belonging to access category AC. AIFSD[AC] is determined by

$$
\text { AIFSD }[A C]=S I F S+\text { AIFS }[A C] \cdot \text { SlotTime, }
$$

where AIFS[AC] is an integer greater than zero. Fig. 3 shows the timing diagram of the EDCF channel access.
The values of AIFS[AC], CWmin[AC], and CWmax[AC], which are referred to as the EDCF parameters, can be determined and announced by the AP via beacon frames, which are transmitted periodically, say every 100 msec typically. The AP can adapt these parameters dynamically depending on network conditions. Basically, the smaller AIFS[AC] and CWmin[AC], the shorter the channel access delay, and hence the more bandwidth share for a given traffic condition. These parameters can be used in order to differentiate the channel access among different priority traffic.

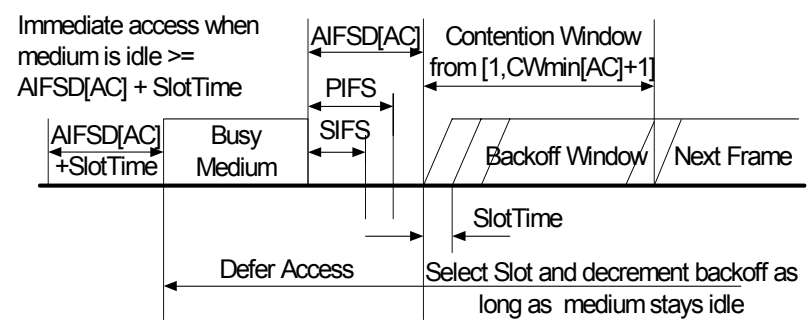

Fig. 3. IEEE 802.11e EDCF channel access.

Fig. 4 shows the 802.11e MAC with four transmission queues, where each queue behaves as a single enhanced DCF contending entity, i.e., an AC, where each queue has its own AIFS and maintains its own Backoff Counter (BC). When there is more than one AC finishing the backoff at the same time, the collision is handled in a virtual manner. That is, the highest priority frame among the colliding frames is chosen and transmitted, and the others perform a backoff with increased CW values.

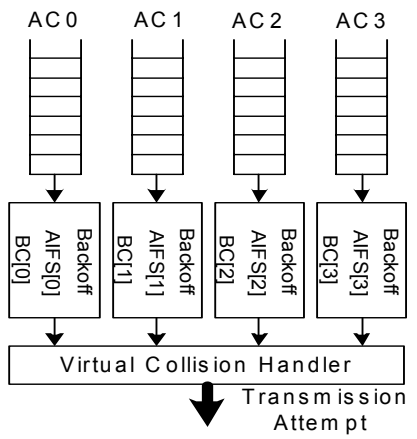

Fig. 4. Four Access Categories (ACs) for EDCF

\section{QoS-Enabled Wired LAN via $802.1 D / Q$}

The IEEE 802.1D MAC bridge specification allows different MAC layers in the IEEE 802 family to interwork. Note that the 802.11 AP typically implements an $802.1 \mathrm{D}$ bridge connecting the 802.11 MAC and 802.3 (or Ethernet) MAC. The $802.1 \mathrm{D}$ bridge supports up to 8 user priorities by implementing multiple FIFO transmission queues between two MAC entities. By default, a priority queuing can be used for these multiple queues. That is, a frame can be forwarded to the egress MAC only if there is no frame in the higher priority queues. As shown in Fig. 5 and Fig. 6, the 802.1Q Virtual LAN (VLAN) tag extends the existing 802.3 frame format, and it specifies the user priority of the frame. Note that the 802.3 MAC itself does not support any differentiated channel access to different priority traffic, but via the $802.1 \mathrm{Q}$ VLAN tag, the 802.3 MAC frames can carry the corresponding priority value, which in turn can be used by the 802.1D MAC bridge for 
a prioritized forwarding. Since the 802.11e EDCF QoS scheme roots in $802.1 \mathrm{D}$, priority parameters of $802.11 \mathrm{e}$ and $802.1 \mathrm{D}$ are interoperable. The User Priority shown in Fig. 6 can be used for TCID valued that is defined in TABLE II.

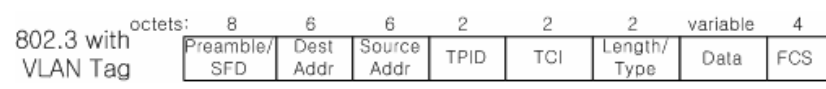

Fig. 5. IEEE 802.3 frame format with 802.1Q VLAN tag

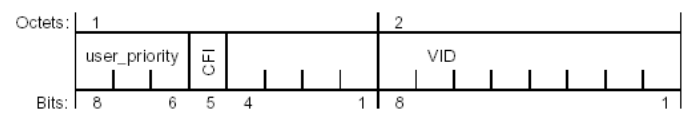

Fig. 6. Tag Control Information (TCI) format within VLAN tag

TABLE II. 802.1D TRAFFIC TYPE [4]

\begin{tabular}{ccc}
\hline User Priority & Acronym & Traffic Type \\
\hline 1 & BK & Background \\
2 & - & Spare \\
0 (Default) & BE & Best Effort \\
3 & EE & Excellent Effort \\
4 & CL & Controlled Load \\
5 & VI & Video $<100$ ms latency and jitter \\
6 & VO & Voice $<10$ ms latency and jitter \\
7 & NC & Network Control \\
\hline
\end{tabular}

When the 802.11e MAC frame is received at the ingress of the VLAN bridge supporting $802.1 \mathrm{D} / \mathrm{Q}$, it is classified by VLAN ID, filtered via filtering ID (FID), and forwarded based on the traffic class that is mapped by user priority [5]. When the traffic class is mapped by user priority, $802.1 \mathrm{D} / \mathrm{Q}$ frames are allocated into specific priority queues according to the traffic classes. When the traffic frames are dequeued from the forwarding process, it is transmitted to the next bridge via egress. 802.1Q [5] has a recommended mapping table between user priorities that are defined in 3 bits of TCI in $802.1 Q$, and traffic classes that specify the priority queue in traffic forwarding process in VLAN Bridge.

\section{END-TO-END QOS COORDINATION BASED DIFFSERV}

It is typical that a single STA simultaneously services multiple sessions under different applications such as VoIP, streaming video, email or FTP. According to the service type, traffic should be differently treated at the network node. As depicted in Fig. 1, three network interfaces were defined in our study as an end-to-end network. Each network interface has independent QoS coordination functions. However, the DSCP is a single point of traffic control across multiple network interfaces so that the end-to-end QoS can be transparently provided over all networks.

\section{A. Over the Air}

In the wireless network, STA performs the packet classification and conditioning in the network layer and forwards the packet to the AP. As illustrated in Fig. 1, a STA should map QoS in IP layer to the 802.11e. In STA supporting DiffServ and 802.11e, the DSCP value should be mapped to the TCID placed in 802.11e MAC QoS Control field. TABLE III depicts an example mapping between DSCP and TCID. DSCP values are recommended by standards [11][12]. According to the traffic control structure, two QoS architectures can be considered as follows: Direct mapped QoS between DSCP and TCID and Hierarchical QoS architecture.
TABLE III. QOS MAPPING TABLE BETWEEN DSCP AND TCID

\begin{tabular}{cccc}
\hline Traffic Class & Example & DSCP & TCID \\
\hline Class 1 & VoIP & $\begin{array}{c}(101) \times x x \text { for } \\
\text { EF }\end{array}$ & 7 \\
Class 2 & Video Streaming & $\begin{array}{c}(100) \times x x \\
(A F 4 x)\end{array}$ & 5 \\
Class 3 & Signaling bearer & $\begin{array}{c}(010) \times x x \\
(A F 2 x)\end{array}$ & 3 \\
Class 4 & $\begin{array}{c}\text { Normal Data } \\
\text { service (e.g. } \\
\text { Web, E-mail) }\end{array}$ & $\begin{array}{c}(000) 000 \\
\text { default best- } \\
\text { effort delivery }\end{array}$ & 1 \\
\hline
\end{tabular}

Direct mapped OoS between DSCP and TCID: This architecture might be simple to map between DSCP and TCID via interface between IEEE 802.2 Logical Link Control (LLC) ${ }^{1}$ Service Access Point (SAP) and PHB. In this model, every IP packet will be placed into $802.11 \mathrm{e}$ MAC priority queues with no preemption. As illustrated in Fig. 7, IP packets are arrived to MAC layer with nonpreemptive mode. Fig. 7 shows that regardless the DSCP values of IP packets, IP packets are forwarded to the 802.11e MAC layer according to the arrival times, which are in order of AF2 (1), AF4 (2), EF (3), and default (4). When the IP packets are encapsulated in MAC frames, each frame should be allocated to a priority queue, or an $A C$, in MAC layer according to its TCID value. Since the TCID field of 802.11e MAC is 3-bit long and the DSCP field of DiffServ is 6-bit long, a single TCID value may represent multiple DSCP values. Due to the different QoS field lengths, the granularity of traffic control should conform to the $802.11 \mathrm{e}$ MAC.

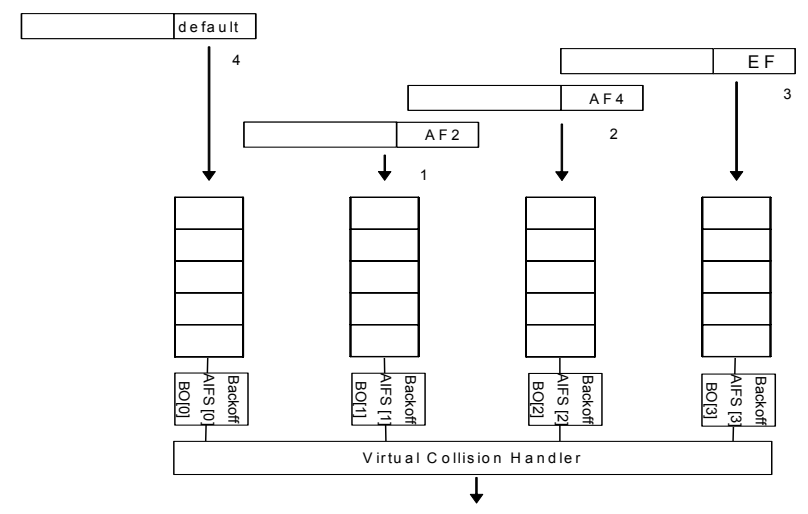

Fig. 7. Direct mapping between DiffServ and 802.11e

Hierarchical $Q O S$ from DSCP and TCID: This architecture uses hierarchical architecture from PHB to the 802.11e Prioritized QoS. The DiffServ engine is a logical entity that performs packet classification and conditioning in the network layer.

As illustrated in Fig. 8, when the IP packets arrive at the DiffServ engine, called Traffic Conditioner (TC), which consists of Classifier, Meter, Marker and Shaper/Dropper, they are classified, marked into DSCP values, and shaped in accordance with the priority of the DSCP values. When DiffServ TC completes the traffic shaping, it encapsulates IP packets into 802.11e MAC frames, and forwards them to $802.11 \mathrm{e}$ priority queues in accordance with the TCID values. For example, as shown in Fig. 8, when the IP packets arrive at DiffServ TC in order of DSCP values, AF4, AF2, EF and default, they are shaped to EF, AF4, AF2 and default according to the

\footnotetext{
${ }^{1}$ In the 802 LAN devices, the 802.2 LLC sits on top of the MAC.
} 
priority. After completing the traffic shaping, IP packets are encapsulated in 802.11e MAC frame and placed into the $802.11 \mathrm{e}$ priority queue.

In this architecture, since IP packets are policed and shaped in the network layer, traffic control can support full range of DiffServ QoS as well as 802.11e. This enables the network system to manage accurate end-toend QoS traffic control required by user applications.

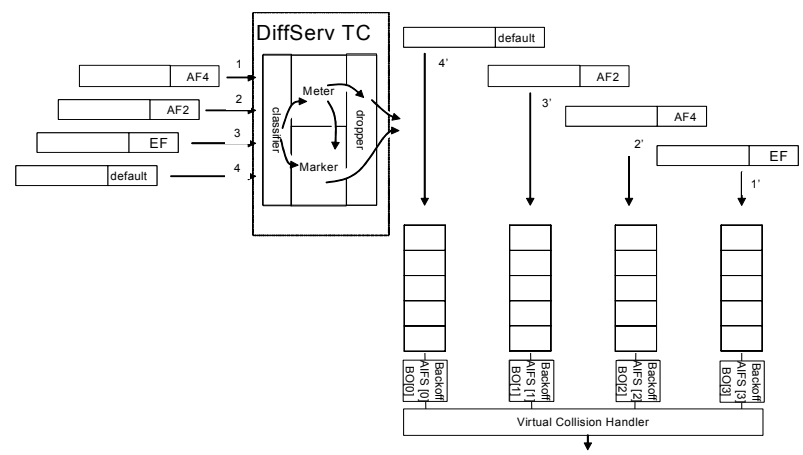

Fig. 8. Combined QoS of DiffServ and 802.11e

\section{B. Ethernet Local Area Network}

As illustrated in Fig. 1, when the AP receives either an Ethernet (i.e., IEEE 802.1D/Q) or a WLAN (i.e., IEEE 802.11e) frame in the local area network, the 802.11e AP shall convert the Ethernet frame into the 802.11e frame, and vice versa. Since User Priority in $802.1 \mathrm{D} / \mathrm{Q}$ and TCID in 802.11e have the identical field size and meaning, they can seamlessly coordinate the QoS parameters. That is, when the $802.11 \mathrm{e}$ prioritized QoS service is used, the first three bits of the 802.1Q TCI field is conveyed in the TCID field of the 802.11e QoS Control field. Note that both sets of three bits indicate the priority value of the frame. Further, the TCID of 802.11e in AP should be coordinated with the User Priorities specified in IEEE 802.1D MAC bridge standard depicted in TABLE II.

\section{Managed IP Wide Area Network}

When the 802.3 MAC frames are terminated at a gateway, as illustrated in Fig. 1, IP packets are reassembled and forwarded to the destination. When the IP packets arrive at the intermediate IP router supporting DiffServ, PHBs are enforced based on the DSCP values. Delay sensitive IP packets in EF class are entered in high priority queue and forwarded with preemption as specified in RFC 2598 [11]. When the IP packets in AF class enter in the IP router supporting QoS, DiffServ engine performs AF PHB. Each AF class (e.g. AF1x, AF $2 x, A F 3 x$ and AF4x) allocates different forwarding resources, which are typically priority buffer size and bandwidth. Once the IP router experiences the traffic congestion, IP packets with AF class will be determined whether or not to be dropped with accordance to the drop precedence values that represents ' $x$ ' above. When the network congestion occurs, IP packets in EF class are always protected for low-jitter, low-loss and low-latency that are defined in SLA. When the SLA defines the QoS traffic control requirements, network administration should configure every IP router under the DiffServ Domain where a single QoS framework is managed. In case of multiple DiffServ Domains, DSCP values can be modified at the Ingress node where the IP packets arrive across network service area.

\section{CONCLUSION}

This paper has presented an end-to-end network QoS architecture engaged with IEEE 802.11e MAC, which is an emerging QoS standard accompanying with the IEEE 802.11 Wireless Local Area Network (WLAN) standard. Each transport level QoS scheme is presented with associated network interfaces, including DiffServ in network and 802.11e and 802.1D/Q in link layer. From the study, it is realized that every QoS scheme has three distinctive processes, which are traffic classification, marking and forwarding. Based on this commonality, end-to-end QoS architecture can be defined with minimal coordination amongst QoS traffic parameters such as DSCP in DiffServ, TCID in 802.11e MAC, and User Priority of TCI in $802.1 \mathrm{D} / \mathrm{Q}$.

It should be emphasized that different QoS granularity between DiffServ and 802.11e should be resolved by hierarchical QoS structure using two-level priority queues. This enables the service providers offering multimedia service via WLAN for their subscribers to manage sophisticated QoS over the end-to-end network. Performance verification remains as a future work.

It should be noted that the 802.11e supports a parameterized QoS paradigm along with the prioritized QoS paradigm considered in this paper [3]. The parameterized QoS characterizes the QoS level with a set of parameters. It will be our future research to study the DiffServ with accordance to the parameterized QoS of the 802.11e.

\section{REFERENCES}

[1] IEEE Std. 802.11-1999, Part 11: Wireless LAN Medium Access Control (MAC) and Physical Layer (PHY) specifications, Reference number ISO/IEC 8802-11:1999(E), IEEE Std. 802.11, 1999 edition, 1999.

[2] IEEE Std. 802.11b, Supplement to Part 11: Wireless LAN Medium Access Control (MAC) and Physical Layer (PHY) specifications: Higher-speed Physical Layer Extension in the $2.4 \mathrm{GHz}$ Band, IEEE Std. 802.11b-1999, 1999.

[3] IEEE 802.11e/D4.0, Draft Supplement to Part 11: Wireless Medium Access Control (MAC) and physical layer (PHY) specifications: Medium Access Control (MAC) Enhancements for Quality of Service (QoS), November 2002.

[4] IEEE 802.1D-1998, Part 3: Media Access Control (MAC) Bridges, ANSI/IEEE Std. 802.1D, 1998 edition, 1998.

[5] IEEE 802.1Q-1998, Virtual Bridge Local Area Networks, ANSI/IEEE Std. 802.1Q, 1998 edition, 1998.

[6] Stefan Mangold, Sunghyun Choi, Peter May, Ole Klein, Guido Hiertz, and Lothar Stibor, "IEEE 802.11e Wireless LAN for Quality of Service," in Proc. European Wireless'02, Florence, Italy, February 2002.

[7] Sunghyun Choi, Javier del Prado, Stefan Mangold, and Sai Shankar, "IEEE 802.11e Contention-Based Channel Access (EDCF) Performance Evaluation," to appear in Proc. IEEE ICC'03, Anchorage, Alaska, USA, May 2003.

[8] Sai Shankar and Sunghyun Choi, "QoS Signaling for Parameterized Traffic in IEEE 802.11e Wireless LANS," Lecture Notes in Computer Science, vol. 2402, pp. 67-84, August 2002.

[9] RFC 2474, Definition of the Differentiated Services Field (DS Field) in the IPv4 and IPv6 Headers, December 1998.

[10] RFC 2475, An Architecture for Differentiated Services, December 1998.

[11] RFC 2598, An Expedited Forwarding PHB, June 1999.

[12] RFC 2597, Assured Forwarding PHB Group, June 1999. 